\title{
Discovery of a magnetic field in the B pulsating system HD 1976
}

\author{
C. Neiner ${ }^{1}$, A. Tkachenko ${ }^{2, \star \star}$, and the MiMeS collaboration \\ 1 LESIA, Observatoire de Paris, CNRS UMR 8109, UPMC, Université Paris Diderot, 5 place Jules Janssen, 92190 Meudon, France \\ e-mail: coralie.neiner@obspm. fr \\ 2 Instituut voor Sterrenkunde, KU Leuven, Celestijnenlaan 200D, 3001 Leuven, Belgium
}

Received 7 February 2014 / Accepted 13 February 2014

\begin{abstract}
Aims. A magnetic field can have a strong impact on the evolution of a binary star. However, only a dozen magnetic OB binaries are known as of today and are available to study this effect, including some very few magnetic pulsating spectroscopic OB binaries. We checked for the presence of a magnetic field in the B5IV hierarchical triple system HD 1976 with spectropolarimetric data obtained with Narval at the Bernard Lyot Telescope (TBL).

Methods. We used orbital parameters of HD 1976 available in the literature to disentangle the Narval intensity spectra. We computed Stokes $V$ profiles with the least squares deconvolution technique to search for magnetic signatures. We then derived an estimate of the longitudinal magnetic field strength for each observation and for various line lists.

Results. Our disentangling of the intensity spectra shows that HD 1976 is a double-lined spectroscopic (SB2) binary, the lines of the secondary component are about twice as broad as those of the primary component. We did not identify the third component. Moreover, we find clear magnetic signatures in the spectropolarimetric measurements of HD 1976 that seem to be associated with the primary component. We conclude that HD 1976 is a magnetic slowly pulsating double-lined spectroscopic binary star with an undetected third component. It is the second such example known (the other is HD 25558).
\end{abstract}

Key words. stars: magnetic field - stars: early-type - binaries: spectroscopic - stars: individual: HD 1976

\section{Introduction}

The magnetic field of OB binaries has not been much explored so far, from either an observational or theoretical point of view, but a magnetic field in a binary might have an important impact on the evolution of the binary.

In low-mass stars, tidal interactions are expected to induce large-scale 3D shear and/or helical flows in stellar interiors that can significantly perturb their magnetic dynamo. Similar flows may also influence the fossil magnetic fields of high-mass stars. Moreover, in combination with tides, magnetically driven winds or outflows in binary systems have long been suspected to be responsible for their orbital evolution, while magnetospheric interactions have been proposed to enhance stellar activity. In addition, the incidence of magnetic stars among OB binary systems provides a basic constraint on the detailed fossil origin of the magnetic field and whether such magnetic fields suppress binary formation. However, the crucial observational constraints required to study these various issues are, at present, nearly nonexistent.

In the frame of the Magnetism in Massive Stars (MiMeS) project (e.g. Neiner et al. 2011; Wade et al. 2013), we searched for a magnetic field in HD 1976.

HD 1976 (V746 Cas) is a bright ( $V=5.6$ ), slowly pulsating B (SPB) star (De Cat et al. 2007; Dubath et al. 2011)

\footnotetext{
* Based on observations obtained at the Telescope Bernard Lyot (USR5026) operated by the Observatoire Midi-Pyrénées, Université de Toulouse (Paul Sabatier), Centre National de la Recherche Scientifique of France.

$\star \star$ Postdoctoral Fellow of the Fund for Scientific Research (FWO), Flanders, Belgium
}

of spectral type B5IV. Its main period of variation is $1.065 \mathrm{~d}$ (HIPPARCos, Perryman et al. 1997). De Cat et al. (2007) and Mathias et al. (2001) found at least one additional period at $2.5 \mathrm{~d}$ and proposed additional candidate periods. HD 1976 seems to be a young star of $\sim 60 \mathrm{Myr}$ (De Cat et al. 2007; Tetzlaff et al. 2011), but note that photometric age determination can lead to uncertainties for multiple systems.

HD 1976 is known to be a hierarchical triple system consisting of a visual binary with a magnitude difference of about 0.9 mag (Perryman et al. 1997; Mason et al. 2009), one of the components is itself an SB1 binary (De Cat et al. 2007). The angular separation of the visual components is 0.157 arcsec according to HIPPARCos (Perryman et al. 1997) and 0.111 arcsec according to Mason et al. (2009). The period of the visual binary is about 150 years (Abt 2005). The SB9 catalogue (Pourbaix et al. 2004) provides a period for the SB1 of between 25.4 and $27.8 \mathrm{~d}$ with an eccentricity of between 0.12 and 0.2 .

In this paper we report on Narval spectropolarimetric observations of HD 1976 (Sect. 2). We use orbital parameters available in the literature to disentangle the spectra of two components (Sect. 3). We then analyse the magnetic measurements with the least squares deconvolution (LSD) technique (Sect. 4) and discuss the results (Sect. 5).

\section{Narval observations}

Narval is a fibre-fed echelle spectropolarimeter with a resolving power of 65000 installed at the Bernard Lyot Telescope (TBL) in France. The spectrograph covers the wavelength domain from 3694 to $10483 \AA$. We collected 16 Stokes $V$ measurements of HD 1976 with Narval between August and October 2012. Their 
Table 1. Log of 16 Narval observations of HD 1976.

\begin{tabular}{llllll}
\hline \hline$\#$ & Date & Mid-HJD & $T_{\text {exp }}$ & $S / N$ & Status \\
& 2012 & -2456000 & $\mathrm{~s}$ & & \\
\hline 1 & 12 Aug. 12 & 152.64640 & $4 \times 900$ & 560 & good \\
2 & 13 Aug. 12 & 153.57493 & $4 \times 900$ & 1010 & good \\
3 & 15 Aug. 12 & 155.58735 & $4 \times 900$ & 620 & good \\
4 & 04 Sep. 12 & 175.53081 & $4 \times 1200$ & 1170 & problem \\
5 & 05 Sep. 12 & 176.56314 & $4 \times 1200$ & 1260 & problem? \\
6 & 06 Sep. 12 & 177.55047 & $4 \times 1200$ & 1280 & problem? \\
7 & 07 Sep. 12 & 178.55895 & $4 \times 1200$ & 1010 & problem \\
8 & 08 Sep. 12 & 179.55751 & $4 \times 1200$ & 1040 & problem? \\
9 & 11 Sep. 12 & 182.58793 & $4 \times 1200$ & 1050 & problem \\
10 & 17 Sep. 12 & 188.48424 & $4 \times 1200$ & 260 & doubtful \\
11 & 19 Sep. 12 & 190.50863 & $4 \times 1200$ & 1260 & doubtful \\
12 & 01 Oct. 12 & 202.48227 & $4 \times 1200$ & 1260 & good \\
13 & 02 Oct. 12 & 203.47914 & $4 \times 1200$ & 1360 & good \\
14 & 03 Oct. 12 & 204.48718 & $4 \times 1200$ & 830 & good \\
15 & 12 Oct. 12 & 213.51685 & $4 \times 1200$ & 760 & good \\
16 & 13 Oct. 12 & 214.39089 & $4 \times 1200$ & 1150 & good \\
\hline
\end{tabular}

Notes. The $\mathrm{S} / \mathrm{N}$ given in col. 5 is the one measured around $5000 \AA$ in the I spectrum. Column 6 indicates the status of the Stokes $V$ measurement related to a technical problem (see Sect. 2).

signal-to-noise ratio (S/N) varies between 260 and 1360 around $5000 \AA$ in the I spectrum (see Table 1). Since the diameter of the Narval fiber is 2.8 arcsec, the light of all three components of HD 1976 has been recorded in the spectra.

During some of our observations, a loss of reference of one of the rhomboedra occurred: the coder was unable to read the exact position of the rhomboedra due to a small wobble of the disk. The lack of reading was compensated for by the closedloop system by changing the speed of the motor, which caused the rhomboedra to jump to an unknown angle (with the coder position kept constant) until the reading resumed. This technical problem does not affect the intensity spectra. It does affect the Stokes $V$ measurements however, as the angle of the rhomboedra was not the one needed to measure the full circular polarisation. When this problem occurred, the Stokes $V$ signature has not been increased, but it could have been decreased or its shape modified, as some of the circularly polarised light was lost. In other words, this problem cannot lead to the appearance of spurious signatures, but it can modify the signatures.

The spectra obtained in August and October are not affected by this technical problem. It only occurred in September. By checking known magnetic stars obtained during the same nights as HD 1976, we found that the spectra obtained on September 4, 7 , and 11 are probably affected by this technical problem. For those obtained on September 5, 6, and 8, there is no magnetic star available to check the reliability of the Stokes $V$ measurements but because they were obtained in the same week as the others, we consider them to be probably affected as well. The two spectra obtained on September 17 and 19 are part of a different observing sequence, but since we have no magnetic check star for these observations either, we consider them to be unreliable. The status of each spectrum is reported in Table 1.

Data were reduced with the LIBRE-ESPRIT reduction package, an extension of ESPRIT (Donati et al. 1997) for Narval available at the telescope. The usual bias and flat-field corrections were applied, as well as a wavelength calibration with a

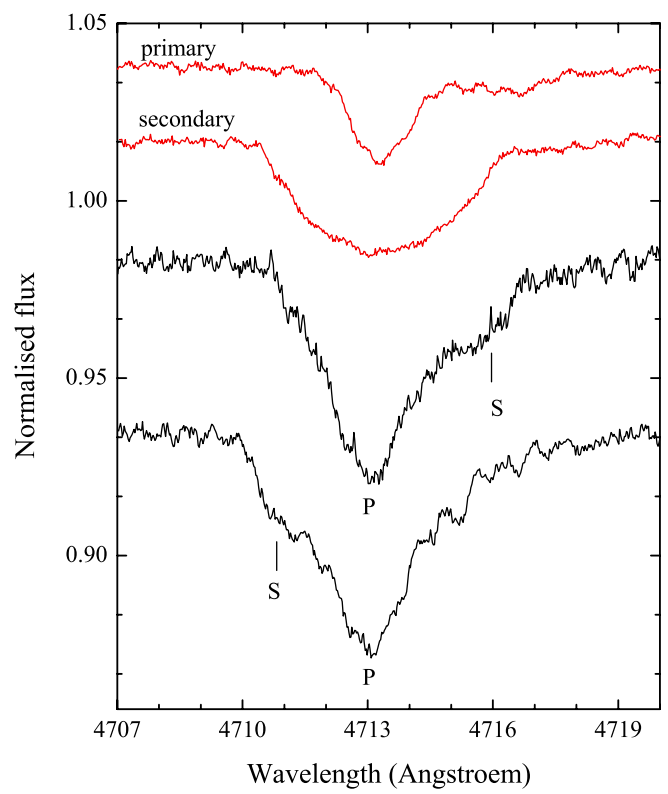

Fig. 1. Bottom (black curves): examples of two spectra (\#1 and \#16) of the He I line at $4713 \AA$ obtained at two different phases of the orbital period. The two binary components are indicated ( $\mathrm{P}$ and $\mathrm{S}$ ). Top (red curves): disentangled spectra of the $\mathrm{He}$ I line at $4713 \AA$ for the primary and secondary components. All spectra are shifted vertically to allow for a better reading.

ThAr lamp. Each echelle order was then carefully normalised with the continuum package of the IRAF software ${ }^{1}$.

\section{Possible system configurations of HD 1976}

Characteristic asymmetries are detected in several individual lines of He and Mg in the spectra of HD 1976 (see Fig. 1), suggesting a double-lined nature of HD 1976. Similar asymmetries are also seen in the LSD Stokes $I$ profiles (see Sect. 4 below).

To verify whether the signal might be attributed to the components of the SB1 or to the visual binary, we applied the spectral disentangling (SPD) technique to our 16 spectra. For this purpose, we used the FDBINARY code (Ilijic et al. 2004), which relies on the Fourier-based implementation of the SPD method suggested by Hadrava (1995). Compared with the original method proposed by Simon \& Sturm (1994), which applies to the spectra in the wavelength domain, the Fourier-based SPD technique has the advantage of being much faster. This makes the method applicable to time-series of high-resolution spectroscopic data.

No precise orbital solution is available for HD 1976 in the literature. The poor phase coverage and limited number of measurements we have at our disposal do not allow us to determine orbital elements either. Therefore, we used all three orbital periods available in the SB9 catalogue (Pourbaix et al. 2004): $25.4176,25.44$, and $27.8 \mathrm{~d}$. We ran the FDBINARY code in pureseparation mode (without optimising for the light factors), focusing on wavelength regions centred on several strong He I (5016 and $4713 \AA), M g$ II (4481 $)$ ) and C II (4267 ̊) lines.

We obtained consistent results for all considered spectral regions and values of the orbital period, that is, contribution of two stars were detected in all cases. Figure 1 illustrates two observed composite spectra (\#1 and \#16) and the disentangled spectra of

1 IRAF is distributed by the NOAO, which is operated by the AURA under cooperative agreement with the NSF. 


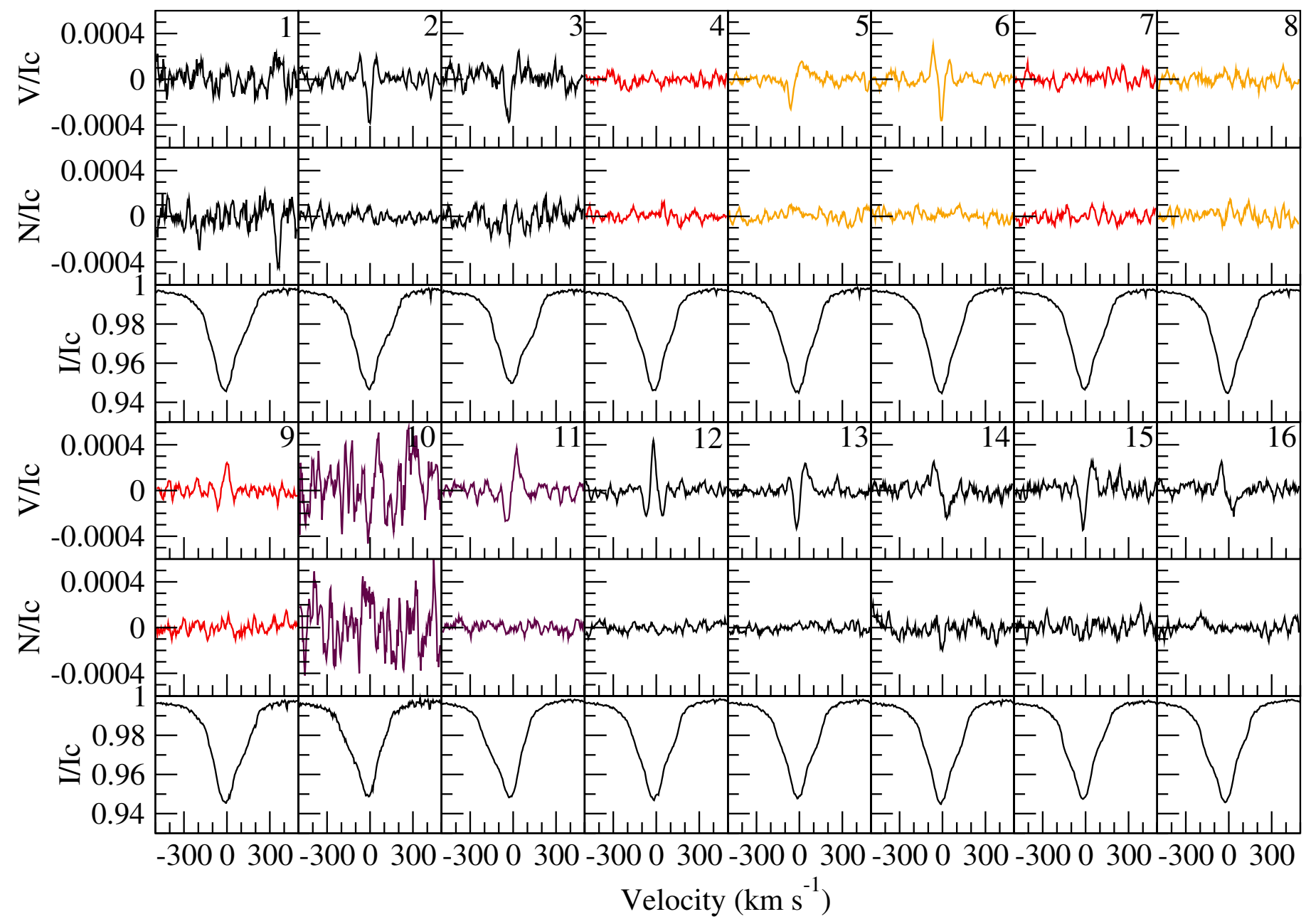

Fig. 2. LSD Stokes $V, N$ and $I$ profiles computed for the composite spectra of HD 1976 with the mask containing all lines. The spectra affected by a technical problem are shown in red, those that are probably affected in orange, those that are doubful in maroon, those without a problem are in black. The reference number of the spectrum is indicated in the corner of each Stokes $V$ panel.

the primary and secondary components. The line profile of the secondary component spans about $374 \mathrm{~km} \mathrm{~s}^{-1}$ and varies significantly in radial velocity, while the profile of the primary spans only about $184 \mathrm{~km} \mathrm{~s}^{-1}$ and is relatively stable in radial velocity. Therefore, the secondary component is clearly associated with the SB1, and the primary component might either be the other component of the SB1 (with a high mass ratio between the two stars) or the visual component, or the sum of both.

We conclude that HD 1976 is a double-lined spectroscopic binary (SB2) star with a spectroscopically undetected third component, but more data are required to obtain a precise orbital solution and identification of the various components in the spectra, as well as the disentangled spectra suitable for detailed spectrum and magnetic analysis of the individual components.

\section{Spectropolarimetric analysis}

We applied the LSD technique (Donati et al. 1997) to the Narval data. To this aim, we first constructed a spectral line list from the VALD database (Piskunov et al. 1995; Kupka et al. 1999) with $T_{\text {eff }}=16000 \mathrm{~K}$ and $\log g=4.0 \mathrm{dex}$ (Mathias et al. 2001). The list only contains lines with a depth higher than $1 \%$ of the continuum level. From this line template we removed all hydrogen lines, lines that were blended with $\mathrm{H}$ lines or interstellar bands, and lines that did not seem to be present in the Narval spectra. We then adjusted the strength of the remaining lines to fit the Narval observations. This resulted in a mask containing 121 lines. From this initial mask, we then also created two submasks containing only the 18 unblended He lines or 85 metal lines except for He lines and lines blended with He.

Using these three line masks, we extracted LSD Stokes $I$ and $V$ profiles for each spectropolarimetric measurements. We also extracted null $(N)$ polarisation profiles to check for spurious signatures, for instance from instrumental origin or stellar pulsations. For the mask with only He lines the mean wavelength is $4832 \AA$ and the mean Landé factor is 1.127 , for the mask without He lines these parameters are $5029 \AA$ and 1.152 , for the mask with all lines they are $4985 \AA$ and 1.196 .

We find that the LSD Stokes $V$ profiles show clear signatures of a magnetic field. In particular, all Stokes $V$ profiles obtained during good conditions (good $\mathrm{S} / \mathrm{N}$ and no technical problem) show a clear magnetic signature (see the first and fourth rows of Fig. 2). In contrast, the $N$ profiles do not show signatures, which confirms that the magnetic measurements have not been contamined by the stellar pulsations, binary or instrumental polarisation effects (see the second and fifth rows of Fig. 2).

The magnetic signatures are observed over a velocity range that spans about $200 \mathrm{~km} \mathrm{~s}^{-1}$ and remain stable in radial velocity, therefore they seem to be associated with the primary component for which the line profiles also span this velocity range and 
Table 2. Longitudinal magnetic field measurements of HD 1976 measured in the composite spectra with three different line masks.

\begin{tabular}{lllllll}
\hline \hline$\#$ & All & & He lines & \multicolumn{3}{c}{ All but He } \\
& $B_{1}$ & $\sigma_{B_{1}}$ & $B_{1}$ & $\sigma_{B_{1}}$ & $B_{1}$ & $\sigma_{B_{1}}$ \\
& $\mathrm{G}$ & $\mathrm{G}$ & $\mathrm{G}$ & $\mathrm{G}$ & $\mathrm{G}$ & $\mathrm{G}$ \\
\hline 1 & 4.4 & 75.1 & 191.6 & 106.8 & -34.0 & 267.7 \\
2 & 22.8 & 33.2 & 44.4 & 46.0 & -67.9 & 106.1 \\
3 & -149.2 & 54.8 & -164.6 & 78.5 & -198.1 & 169.7 \\
$(4)$ & 7.1 & 28.9 & -24.4 & 41.4 & 81.6 & 80.7 \\
$(5)$ & -153.5 & 25.3 & -111.5 & 35.5 & -407.1 & 83.0 \\
$(6)$ & 56.7 & 25.2 & 41.8 & 35.0 & 88.5 & 77.4 \\
$(7)$ & 11.2 & 33.3 & -55.9 & 46.9 & 44.1 & 97.5 \\
$(8)$ & -12.5 & 31.4 & -58.9 & 43.8 & 137.9 & 101.0 \\
$(9)$ & 3.5 & 31.0 & 46.1 & 43.5 & -69.4 & 113.0 \\
$(10)$ & -91.7 & 163.3 & -213.8 & 262.1 & 100.8 & 410.5 \\
$(11)$ & -208.1 & 27.6 & -222.3 & 39.1 & -292.4 & 74.7 \\
12 & 5.6 & 26.5 & -65.1 & 36.7 & 50.5 & 85.0 \\
13 & -83.0 & 25.5 & -11.4 & 35.4 & -296.3 & 72.1 \\
14 & 206.4 & 39.6 & 132.2 & 55.2 & 554.7 & 127.6 \\
15 & -107.1 & 44.7 & -111.9 & 63.8 & -153.3 & 123.7 \\
16 & 156.1 & 28.5 & 134.3 & 39.7 & 352.5 & 91.7 \\
\hline
\end{tabular}

Notes. Measurements from \#5 to \#11 indicated in brackets should be considered with care because of a technical problem with the polarimeter (see Sect. 2 and Table 1).

which does not vary much in radial velocity. However, we cannot exclude that the secondary component is also magnetic (with a weaker field) without an accurate disentangling of the spectra.

The longitudinal field $\left(B_{1}\right)$ values and their error bars $\left(\sigma_{B_{1}}\right)$, extracted from the LSD Stokes $V$ profiles in the range from -120 to $120 \mathrm{~km} \mathrm{~s}^{-1}$ using each mask, are reported in Table 2 . However, since these values were extracted with the composite I spectrum of the two components of HD 1976, they do not represent the real field values of the magnetic component but an underestimate. We also recall that the values extracted in certain nights might be affected by a technical problem with the rhomboedra position (see Sect. 2). These $B_{1}$ values therefore need to be considered with care and are marked in Table 2.

\section{Discussion}

Using orbital parameters available in the SB9 catalogue (Pourbaix et al. 2004), we have been able to disentangle the spectra of two components of HD 1976. This shows that HD 1976 is an SB2 binary. The primary component has a lower $v \sin i$ value $\left(\sim 90 \mathrm{~km} \mathrm{~s}^{-1}\right)$ than the secondary component $\left(\sim 190 \mathrm{~km} \mathrm{~s}^{-1}\right)$. The third component remains hidden in our spectra.

However, various orbital periods are proposed in the literature and we were unable to distinguish between them or identify the origin of the primary component precisely. Therefore our disentangled spectra are too uncertain to be used to compute reliable magnetic measurements of the individual components.

Nevertheless, the Narval spectropolarimetric measurements clearly show that HD 1976 is a magnetic star. The magnetic signatures seem to be associated with the primary component.

The longitudinal magnetic field measured using all lines varies between about -200 and $200 \mathrm{G}$. These values are underestimated for two reasons however: first we used the composite intensity spectra to derive them and thus the Stokes $V$ spectra were normalised with a too strong intensity, second a technical problem with the polarimeter rhomboedra led to a loss of part of the circular polarisation signal for some of the measurements.
Assuming an oblique dipole field, as observed in most massive stars and as suggested by the shape of the observed Stokes $V$ profiles (see Fig. 2), and following Schwarzschild (1950), we estimate the polar field strength to be of at least $600 \mathrm{G}$ according to the above longitudinal field values. Considering that these values are underestimated, the polar field might be much stronger.

\section{Conclusions}

HD 1976 is a very interesting object because known magnetic pulsating spectroscopic binary are rather rare. The only other known SPB example is HD 25558 (Sódor et al. 2014).

We plan on acquiring new spectra of HD 1976 to derive a more precise orbital solution. We will then be able to disentangle the spectra more reliably and measure the magnetic field of the individual components. This is necessary to determine whether the secondary component is also magnetic, and to derive more accurate measurements of the magnetic field of the primary. Moreover, we will be able to test whether the magnetic component is part of the SB1 and is the pulsating component.

Finally, the Binarity and Magnetic Interactions in various classes of stars (BinaMIcS) project (see Neiner et al. 2013), which relies on two large observing programs with ESPaDOnS at CFHT and Narval at TBL, will allow us to acquire spectropolarimetric observations of many magnetic binary systems of all spectral types. These new observations will provide additional examples of magnetic massive binary stars, in particular systems with short orbital periods. Comparing HD 1976 with other magnetic massive binaries will be very useful to derive information about the role of magnetic field on binarity and vice-versa.

Acknowledgements. C.N. wishes to thank the Programme National de Physique Stellaire (PNPS) for their support. The research leading to these results has partly received funding from the Fund for Scientific Research of Flanders (FWO), Belgium, under grant agreement G.0B69.13. This research has made use of the SIMBAD database operated at CDS, Strasbourg (France), and of NASA's Astrophysics Data System (ADS).

\section{References}

Abt, H. A. 2005, ApJ, 629, 507

De Cat, P., Briquet, M., Aerts, C., et al. 2007, A\&A, 463, 243

Donati, J.-F., Semel, M., Carter, B. D., Rees, D. E., \& Collier Cameron, A. 1997, MNRAS, 291, 658

Dubath, P., Rimoldini, L., Süveges, M., et al. 2011, MNRAS, 414, 2602

Hadrava, P. 1995, A\&AS, 114, 393

Ilijic, S., Hensberge, H., Pavlovski, K., \& Freyhammer, L. M. 2004, in Spectroscopically and Spatially Resolving the Components of the Close Binary Stars, eds. R. W. Hilditch, H. Hensberge, \& K. Pavlovski, ASP Conf. Ser., 318, 111

Kupka, F., Piskunov, N., Ryabchikova, T. A., Stempels, H. C., \& Weiss, W. W. 1999, A\&AS, 138, 119

Mason, B. D., Hartkopf, W. I., Gies, D. R., Henry, T. J., \& Helsel, J. W. 2009, AJ, 137, 3358

Mathias, P., Aerts, C., Briquet, M., et al. 2001, A\&A, 379, 905

Neiner, C., Alecian, E., \& Mathis, S. 2011, in SF2A-2011: Proc. Annual meeting of the French Society of Astronomy and Astrophysics, eds. G. Alecian, K. Belkacem, R. Samadi, \& D. Valls-Gabaud, 509

Neiner, C., Alecian, E., \& the BinaMIcS Collaboration 2013, EAS Publ. Ser., 64, 75

Perryman, M. A. C., Lindegren, L., Kovalevsky, J., et al. 1997, A\&A, 323, L49

Piskunov, N. E., Kupka, F., Ryabchikova, T. A., Weiss, W. W., \& Jeffery, C. S. 1995, A\&AS, 112, 525

Pourbaix, D., Tokovinin, A. A., Batten, A. H., et al. 2004, A\&A, 424, 727

Schwarzschild, M. 1950, ApJ, 112, 222

Simon, K. P., \& Sturm, E. 1994, A\&A, 281, 286

Sódor, Á., De Cat, P., Wright, D. J., et al. 2014, MNRAS, 438, 3535

Tetzlaff, N., Neuhäuser, R., \& Hohle, M. M. 2011, MNRAS, 410, 190

Wade, G. A., Grunhut, J., Alecian, E., et al. 2013 [arXiv: 1310. 3965] 